
\title{
3 Research Square \\ Factors that impact the adoption of clinical decision support systems (CDSS) in healthcare settings for evidence-based decision making
}

\section{MAH LAKA}

The University of Adelaide https://orcid.org/0000-0003-3228-5031

\section{ADRIANA MILAZZO}

The University of Adelaide

TRACY MERLIN ( $\square$ tracy.merlin@adelaide.edu.au )

https://orcid.org/0000-0002-7293-4262

\section{Research article}

Keywords: clinical decision support tools; barriers; facilitators; UTAUT

Posted Date: June 22nd, 2020

DOI: https://doi.org/10.21203/rs.3.rs-34358/v1

License: (c) (i) This work is licensed under a Creative Commons Attribution 4.0 International License.

Read Full License 


\section{Abstract}

Background: Clinical decision support systems (CDSS) are designed to promote evidence-based patient care and shared-decision making in healthcare settings. Despite these benefits, adoption and long-term use of the systems remain limited. There is a need to identify different factors that influence CDSS adoption in healthcare settings.

Objective: The Unified Theory of Acceptance and Use of Technology (UTAUT) model was applied to determine different factors that influence the adoption of CDSS in healthcare settings.

Methods. A cross-sectional online survey examining clinicians' perceptions about CDSS implementation in hospital and primary care settings in Australia was undertaken from June - October 2019. Multivariate logistic regression was used to examine the association of UTAUT moderators (age, gender and experience) and care settings (hospital and primary care) with perceived benefits, barriers and facilitators to CDSS adoption.

Results: Access to information required for evidence-based decision making, and improvements in quality and safety of patient care were the most common perceived benefits of CDSS. Mapping of different barriers and facilitators to the UTAUT model indicated that ease of use (effort expectancy), perceived benefit (performance expectancy) and a facilitating environment greatly influence the adoption of CDSS. Respondents indicated that systems providing a better fit between relevance, content and timeliness greatly facilitates uptake. Adoption of CDSS also depends on the ability of an organisation to create a facilitating environment that can help address the lack of users' trust in these systems. The type of healthcare setting was found to be a significant predictor of lack of confidence in the content within CDSS, threat to professional autonomy, and time constraints as barriers to CDSS implementation. Therefore, setting dynamics as well as user-specific requirements need to be considered to improve the acceptability and use of CDSS.

Conclusion: Our study has explored different factors that may help address implementation challenges for CDSS. By combining internal factors of users' inclination and perceptions about the system's perceived benefits, coupled with external factors of system design requirements, training and support, and stakeholders' consultations, our findings highlight the need for a holistic implementation framework to enable effective CDSS adoption.

\section{Introduction}

Antibiotic resistance has been recognised as one of five major global health threats. It is estimated that by 2050 drug-resistant infections will lead to 10 million deaths worldwide [1]. Inappropriate antibiotic prescribing has been identified as a major driver to increasing antibiotic resistance [2]. Standardising clinical practices, improving quality and safety of care and reducing inappropriate prescribing have become the top priorities for antimicrobial stewardship programs. Digital health technology tools are 
quickly becoming an essential part of the healthcare system due to the increasing need to deliver valuebased care [3, 4].

Clinical decision support systems are systems that provide clinicians with information for point of care decision making. By connecting evidence-based information on appropriate prescribing (a knowledgebase) with patient information, these systems filter and present the right information at right time to clinicians to assist their decision making and prescribing behaviour $[5,6]$. Benefits of CDSS for antibiotic stewardship include optimising the prescribing process by allowing feedback and audit as well as increasing compliance with antibiotic prescribing guidelines $[7,8]$.

Despite increasing evidence regarding CDSS benefits, the adoption and uptake of CDSS by clinicians (the end-users) remains very limited [9]. Most of the research that has been carried out at the intersection of CDSS and antibiotic management tend to focus on the systems' technical design. The research has overlooked the fact that success of the digital system is equally dependent on end-users inclination to use the system [10]. Previous studies show that CDSS adoption is far less than anticipated with $96 \%$ of alerts or recommendations ignored [11, 12]. Implementation of these systems can be very challenging due to the complex interaction of system, organisational and human factors. Given this, a socio-technical assessment of the implementation process may identify barriers in the adoption and use of CDSS. Our study aim was to identify different factors at the individual, organisational and system level that influence the adoption and use of CDSS. Evaluation of the perceptions and insights of clinicians regarding enablers and barriers to CDSS implementation will enhance an understanding of non-technical factors that may influence the long-term successful implementation of these systems.

\section{Methodology}

\section{Theoretical Framework}

We have used the Unified Theory of Acceptance and Use of Technology (UTAUT) model to understand the interplay between different organisational, individual and technical factors that may influence the successful adoption and use of CDSS [13]. Four basic constructs of this model, namely effort and performance expectancy, social or environmental influence and enabling conditions, helped us to determine specific factors related to users' intentions to adopt a CDSS. The UTAUT integrates a range of theoretical models to provide a comprehensive framework for investigating user intention and acceptance of a technology [14]. Fig. 1 provides the theoretical framework utilised in this study.

Fig. 1 Theoretical framework (UTAUT model) of the study

\section{Study Design}


This study employed a cross-sectional descriptive design. An online survey of hospital and primary care clinicians (physicians, surgeons and general practitioners) across Australia was administered from June October 2019. Recruitment was assisted by the Royal Australian Colleges of Physicians, General Practitioners and Surgeons as well as local health networks across Australia. These organisations promoted the survey via their newsletters, websites, networks, and social media accounts.

\section{Study Participants}

The target population for this survey was the clinical workforce, specifically physicians, surgeons and general practitioners in Australia who are directly involved in antibiotic prescribing. The sampling framework was established using information from the National Health Workforce Data Set comprising medical practitioner data (2011 - 2015). We estimated a sample size of 350 clinicians from primary care, and 350 from hospital settings in order to generalise the survey results to the Australian medical practitioner population. The power required for this study was established using Cochran's sampling method with $95 \%$ confidence interval and $5 \%$ margin of error.

\section{Questionnaire instrument}

The survey questions were designed following an extensive literature review of similar studies [15-20]. The questionnaire comprised of 15 questions covering four sections: demographics; availability of electronic prescribing systems and the perceived impact; and barrier and facilitators for use of CDSS. The purpose of the survey was to identify factors that influence the adoption and acceptance of CDSS in healthcare settings. Questions about the clinicians' demographic characteristics and availability of electronic prescribing systems in respective practices were included to evaluate potential differences in system adoption that might be related to these characteristics. Questions regarding the perceived benefit, barriers and facilitators of CDSS could be answered using five point Likert-type scales, where 1 represented "Strongly Disagree"; $2=$ "Disagree"; $3=$ "Neither Agree Nor Disagree"; $4=$ "Agree" and 5= "Strongly Agree". Most of the survey questions were closed; however, an open-ended question and comments section were also included in the survey to allow respondents to provide additional comments. These responses were then analysed using Braun and Clarke's (2006) thematic analysis process. All the similar codes were grouped into themes which were reviewed iteratively for the validity of each theme.

The survey was conducted online through SurveyMonkey TM (www. surveymonkey.com, San Mateo, California, US). The online survey was initially pilot tested with known clinical contacts $(n=10)$ to evaluate its acceptability by a clinical workforce before it was distributed widely. After reviewing the results in the pilot phase modifications were made by the research team to the survey's text.

\section{Measures}


As it was not mandatory for participants to provide a response to all questions, the number of responses for each question was calculated separately. The information on demographic characteristics and availability of electronic prescribing systems was used to evaluate the impact of these factors on the clinicians' perception regarding CDSS adoption and use. Similarly, moderating factors (age, gender, and experiences) proposed in UTAUT model also helped to investigate how these factors moderate the association between model constructs and the intention to use CDSS.

Dichotomous dependent variables were generated from the Likert 5-point scale for each question by collapsing the responses into binary responses of "Yes" and "No/Unsure" to represent participants' perceptions regarding benefits, barriers, and facilitators for CDSS implementation. The responses of "Agree" and "Strongly Agree" were combined to formulate a binary response of "Yes", whereas "Disagree" and "Strongly Disagree" responses were coded "No/Unsure". The neutral response of "Neither Agree nor Disagree" was also included in the "No/Unsure" category due to low responses and could not be included as a separate category. The rationale of combining neutral with negative responses was that the focus of analysis was identifying participants who have positively responded to the survey questions.

\section{Statistical Analysis}

Bivariate logistic regression analyses were carried out for an exploratory evaluation of the association between binary responses and respondents' characteristics of age, gender, experience, care settings, type of practice and availability of electronic prescribing systems. Associations with a significance level $<0.2$ were included in the multivariate logistic regression model to examine the effect of respondents' characteristics on binary responses. Using backward elimination, variables with a significance level of 0.05 were retained in the final model and included age, experience, and type of care setting. The results are provided as an Odds Ratio (OR) with $95 \%$ confidence interval (Cl).

All the statistical analysis on the data was performed using Stata 15 (StataCorp LP, College Station, TX).

\section{Qualitative Analysis}

The survey incorporated an open-ended question to obtain information on any specific concern(s) participants had about CDSS. The open-ended responses were analysed for contextual content using NVivo 11 (QSR International Pty Ltd, Doncaster, Victoria, Australia). The preliminary codes were generated through open coding of the qualitative data [21]. Using recursive comparison, these codes were then refined and merged into conceptual themes.

\section{Ethical Approval}

Ethical approval was obtained from the University of Adelaide Human Research Ethics Committee (approval number: H-2019-094). The participants could refuse to provide response to any question. 
Participation in the survey was voluntary and the data collected was non-identifiable. To off-set the expected low survey response rates the respondents were given the opportunity to participate in a draw either to win an iPad or equivalent donation will be made in their name to the Hospital Research Foundation in recognition of their participation.

\section{Results}

\section{Response rate}

A total of 180 clinicians participated in the survey with 74 from primary care (general practitioners) (response rate $21.1 \%$ ) and 106 from hospital care (physicians and surgeons) settings (response rate 29.4\%). Our response rate is consistent with many other international and Australian studies conducted with a clinical workforce. The literature reports a $30-70 \%$ response rate; however, lower than $20 \%$ has also been reported [22-25]. A national longitudinal survey of medical professionals in Australia reported an overall response rate of $19.36 \%$, with $17.6 \%$ of GPs replying and $22.3 \%$ of specialists [26]. Missing values for questions ranged from $8.9 \%$ to $16.4 \%$.

\section{Characteristics of study participants}

Participant demographic characteristics are described in Table 1. The majority (66\%) were males and aged between 35 - 54 years (47\%). Most respondents (42\%) had 11-20 years of experience working in the healthcare sector. More respondents (56\%) resided in the eastern states of Australia.

Table 1 Characteristics of study participants 


\begin{tabular}{|c|c|c|}
\hline \multicolumn{2}{|c|}{ Characteristics } & $\mathrm{N}=180(\%)$ \\
\hline \multicolumn{3}{|l|}{ Gender } \\
\hline \multicolumn{2}{|l|}{ Male } & $118(66)$ \\
\hline \multicolumn{2}{|l|}{ Female } & $62(34)$ \\
\hline \multicolumn{3}{|l|}{ Age-group } \\
\hline \multicolumn{2}{|l|}{ 18-34 years } & $61(34)$ \\
\hline \multicolumn{2}{|l|}{$35-54$ years } & $84(47)$ \\
\hline \multicolumn{2}{|c|}{55 years and over } & $35(19)$ \\
\hline \multicolumn{3}{|c|}{ Years of experience } \\
\hline \multicolumn{2}{|l|}{$1-10$ years } & $57(32)$ \\
\hline \multicolumn{2}{|l|}{$11-20$ years } & $75(42)$ \\
\hline \multicolumn{2}{|c|}{ More than 20 years } & $48(27)$ \\
\hline \multicolumn{3}{|c|}{ Care setting and type of practice } \\
\hline \multirow[t]{4}{*}{ Hospital(s) } & Public & $44(24)$ \\
\hline & Private & $14(8)$ \\
\hline & Mixed & $35(19)$ \\
\hline & Total & $93(51)$ \\
\hline \multirow[t]{5}{*}{ Primary care } & Private & $15(8)$ \\
\hline & Community clinic & $11(6)$ \\
\hline & Hospital-based clinic & $12(7)$ \\
\hline & Mixed & $25(14)$ \\
\hline & Total & $63(35)$ \\
\hline \multicolumn{3}{|c|}{ State and territory, Australia ( $n=139)$} \\
\hline \multicolumn{2}{|c|}{ Eastern (ACT/NSW/QId/Tas/Vic) } & $101(73)$ \\
\hline \multicolumn{2}{|c|}{ Central (SA/NT) } & $21(15)$ \\
\hline Western (WA) & & $17(12)$ \\
\hline
\end{tabular}

\section{Perceived benefit of CDSS}


Overall, respondents had access to a variety of electronic systems/modules, with $76 \%$ having patient information management (PIM) systems in their practices, 69\% medication management (EMM) systems, and just over half (52\%) had any form of clinical decision support system (CDSS) available for their use. Of all the respondents who had access to CDSS in their practices, there was a higher proportion accessing them in primary care (62\%) than in hospital settings (38\%).

In terms of perceived benefits, approximately half agreed (48\%) that CDSS can help in reducing the overall cost of antibiotic therapy leading to more cost-effective treatment (Fig. 2). This contrasted with $79 \%$ of respondents who agreed that implementation of CDSS can increase the accessibility to information for optimal decision-making (Fig. 2). However, as shown in Table 2, this latter association was strongly associated with healthcare setting. Clinicians in primary care were $76 \%$ less likely than those in hospital settings to believe that accessibility to protocols and guidelines can be improved through CDSS use $(\mathrm{OR} 0.24,95 \% \mathrm{Cl} 0.09,0.58)$. Clinicians in primary care were half $(\mathrm{OR} 0.54,95 \% \mathrm{Cl} 0.26$, 1.1) as likely as hospital clinicians to believe that CDSS has the potential to improve the overall safety and quality of care, although this varied with respondents' work experience. In comparison to the referent category (1-10 years), respondents with clinical experience of $11-20$ years, and $>20$ years were $56 \%$ and $72 \%$ respectively less likely to believe that CDSS can have any positive impact on quality and safety of care (experience $11-20$ years: OR $0.44,95 \% \mathrm{Cl} 0.19,0.98$; experience $>20$ years: OR $0.28,95 \% \mathrm{Cl} 0.05$, 0.66) (Table 2).

There was variability in responses overall, with several associations having wide confidence intervals.

Fig. 2 Overall perceived benefits of CDSS

Cl: Confidence Interval; OR: Odds ratio

*Significant predictors, as confidence interval does not include 1.0

\section{Perceived Barriers}

Respondents' perceptions regarding different factors that can impact CDSS adoption are shown in Fig. 3 . Of all respondents, $69 \%$ indicated lack of technical knowledge and training are an important barrier for CDSS adoption. On the other hand, $63 \%$ of respondents believed that end users' lack of trust and confidence in the content of the system limits the usability of CDSS. Other frequently identified barriers that may make it difficult for end-users to interact with CDSS effectively concern the dynamics of the clinical environment (Fig. 3). 
As shown in Table 3, type of healthcare setting was significantly associated with clinician's perceptions regarding certain barriers such as time constraints, limits on professional autonomy, and patient preferences toward CDSS. Clinicians in primary care settings were twice as likely as hospital clinicians to believe that time limitations (OR $1.95,95 \% \mathrm{Cl} 1.10,3.77)$, threat to professional autonomy $(\mathrm{OR} 2.15,95 \% \mathrm{Cl}$ $1.39,3.41)$ and patients' preferences (OR 2.17, $95 \% \mathrm{Cl} 1.14,3.37)$ will restrict the use of CDSS. Moreover, the likelihood of perceiving limited professional autonomy as a barrier was also found to increase with clinical experience (11-20 years: OR 1.51, 95\% Cl 1.08, 2.17; > 20 years: OR 1.91, 95\% Cl 1.16, 2.73) (Table 3 ). We found that $71 \%$ of respondents in primary care had clinical experience of $>11$ years compared to respondents in hospital settings (54\%). Therefore, the association of healthcare settings with threat to professional autonomy as a barrier may be related to a higher proportion of experienced respondents in the primary care group. Overall, experienced clinicians were also more likely to believe that lack of confidence in the content of the CDSS would inhibit its use (>20 years OR 1.91, 95\% Cl 1.16, 3.03).

\section{Cl: Confidence Interval; OR: Odds ratio}

*Significant predictors as confidence interval does not include 1.0

Fig. 3 Reported barriers to CDSS adoption.

\section{Perceived Facilitators}

Figure 4 highlights strong agreement (75\%) among respondents that when systems are easy to use this facilitates CDSS adoption whereas $64 \%$ believed that organisational support is required for successful implementation. Along with organisational support, $61 \%$ also agreed that effective training and technical support ensures clinicians receive adequate technological support and skills to use CDSS effectively. Stakeholders' consultation to ensure systems are more adaptable to specific users-requirements was also cited as an important facilitator by $60 \%$ of respondents.

Healthcare setting was identified to be significantly associated with perception of certain factors as enablers to CDSS adoption. Compared to hospital settings, respondents working in primary care settings were $39 \%$ more likely to believe that ease of use will facilitate CDSS adoption (OR 1.39, 95\% Cl 1.15, 2.07). However, the view that technical training will improve adoption and use of CDSS was less likely to be held among primary care respondents $(\mathrm{OR} 0.26,95 \% \mathrm{Cl} 0.11,0.62)$. Clinical experience by length of time was a significant predictor for perceiving stakeholder consultation as an important facilitator to CDSS use. Those with greater than 11 years of experience were more likely to consider stakeholder participation as a facilitator in comparison to reference category of 1-10 years of experience (experience 
11-20 years: OR 1.97, 95\% Cl 1.08, 2.88; experience > 20 years: OR 2.11, 95\%Cl 1.16, 3.04). In contrast, compared to younger clinicians ( 18 - 34 years) those aged $35+$ years were less likely to consider organisational support as an enabling factor for CDSS adoption (age $35-54$ years: OR $0.65,95 \% \mathrm{Cl} 0.26$, 0.91 ; age 55 > years: OR $0.28,95 \% \mathrm{Cl} 0.08,0.54$ ) (Table 4 ).

Fig. 4 Facilitators to CDSS adoption

Cl: Confidence Interval; OR: Odds ratio

*Significant predictors as confidence interval does not include 1.0

\section{Qualitative Analysis}

Analysis of free-text comments provided three major themes related to factors that influence CDSS implementation:

\section{i. Lack of flexibility.}

Respondents expressed concerns regarding CDSS inflexibility to change and cited it as a barrier to CDSS adoption. They reported that system usefulness is significantly limited if it lacks the ability to reflect the complex clinical context by taking into consideration setting and patient-specific factors:

"Systems I have experienced are comically bad in design mainly because they are inflexible in its ability to change "(Hospital)

Clinicians require flexibility and adaptability in systems instead of "constant rule-making" to tailor recommendations to specific context.

"There is never a 'one size fits all'. So there must always be room to make exceptions" (Primary care)

ii. Information overload

"My major frustration with it [CDSS] in terms of antibiotic therapy is the presence of excessive allergy alerts, which do nothing to protect patients and simply lead to alert fatigue." (Hospital)

Information relevance and precision emerged as important factors that influence CDSS adoption. Respondents indicated that excessive information with low specificity and relevancy leads to alert fatigue and override, thereby reducing the overall use of CDSS. Furthermore, it was highlighted that time and workload pressures make it difficult for clinicians to distinguish important information from irrelevant data. 
iii. Information accuracy.

"I, as a user, need to know on what basis any recommendation is provided, what is the source of this knowledge and how often it is updated" (Primary care)

The accuracy of the content was also identified as an important theme for clinicians to trust using CDSS. Respondents expressed doubts concerning the currency and reliability of the content which determines their overall trust in the system.

"...without knowing how often guidelines are updated in the system, we cannot rely on system alerts" (Hospital)

The uncertainty felt by clinicians about the quality and accuracy of evidence negatively impacts their perception of CDSS.

\section{Discussion}

This study highlights clinicians' perception regarding the implementation barriers and facilitators for CDSS. We specifically focused on internal and external factors influencing users' intention to adopt CDSS by incorporating the framework of UTAUT. The internal related factors were specific to personal expectations and perceptions, whereas external factors represented organisational, technical or patient related factors. Our findings indicate a significant interplay between constructs of the UTAUT model namely perceived benefit (performance expectancy), perceived ease of use (effort expectancy) and organisational support (facilitating conditions) for CDSS adoption. Along with investigating relevance of different constructs, the association of moderators (age, gender, experience) and healthcare settings within these constructs was also explored.

\section{Barriers and Facilitators}

\section{External factors}

Our findings highlight that the facilitating environment has a significant impact on the adoption and usage of CDSS. One of the most significant barriers identified was lack of organisational capacity to provide appropriate technical support and training to end users. This can lead to limited understanding of the system's features as well as difficulty to interact with the system effectively [27]. This can also be related to individual user's computer literacy as clinicians who routinely use computers are reported to have better adoption of systems such as CDSS [28]. Nonetheless, lack of support and training can limit the users' confidence in the system and ability to resolve any technical issues that may arise, thus discouraging CDSS adoption [29, 30]. Interestingly, where the lack of organisational support greatly restricts the effective implementation of CDSS, the availability of appropriate infrastructure and supportive organisational environment was cited most frequently among our respondents as a facilitator. 
Different studies that have used organisational theories to explain the technology acceptance behaviour also identify organisational structure and culture as enabling factors for promoting adoption of any new technology [31]. Our results further indicated that young clinicians (aged 18- 34 years) are more likely to require organisational support for adoption of CDSS. This might be related to overall clinical hierarchy and lack of familiarity with the clinical environment. The clinical hierarchy and seniors' preferences greatly influence the perception and practices of young clinicians [32]. Therefore, it is necessary to establish an organisational environment that fosters acceptability, support and trust for CDSS adoption.

One of the basic constructs of UTAUT related to overall system quality is the ease of use of the system. This signifies users' effort expectancy (effort required to use the system). In our survey, ease of use was identified as a key factor that facilitates adoption and adherence of clinicians with the CDSS. This is consistent with the measures of information system (IS) success model proposed by DeLone and Mclean (1992) that relates user satisfaction and adoption to ease of use [33,34]. We found that primary care clinicians perceive ease of use to be the most important feature for adoption of CDSS. These results concur with previous studies on adoption of technology in primary care, and is believed to be related to limited consultation time, workload, computer self-efficacy and patients' expectations in primary care [27, 35]. Due to time constraints and workload pressure, general practitioners may avoid using CDSS due to risk of encountering irrelevant and overwhelming amount of information, as well as compromising on direct communication with patients [36, 37].

Effort expectancy and perceived benefit of the system is related to users' trust that the system is a better fit to their specific requirements. Therefore, more than half of the respondents identified stakeholder consultation in the implementation of CDSS as a facilitator. This is related to identifying end-users' concerns, expectations and workflow requirements. Our results also showed that clinicians with longer working experience tended to rate stakeholders' involvement as more important for facilitating CDSS implementation. This is in agreement with the UTAUT theory that identified years of experience as a mediating factors for users' inclination to adopt technology [38]. Similarly, our results also indicated that clinicians with longer clinical experience (> 11 years) are more likely to see CDSS as a threat to their clinical autonomy. Therefore, inclusion of experienced clinicians in the CDSS development and implementation process would likely foster increased acceptance, trust and compliance with the CDSS.

\section{Internal factors}

In our study, internal factors were frequently reported as barriers to CDSS adoption. The most frequently reported barrier related to CDSS performance expectancy was lack of confidence in the content of the system. Not only is it a result of limited understanding of the system, but also a by-product of other factors concerning lack of trust in personnel involved in development of the system and lack of agreement with the guidelines or content of the system $[39,40]$. Many surveys have indicated that clinicians believe standard guidelines used in these systems usually do not take into consideration context-specific requirements or patient-specific risks, thus reducing their applicability for complex clinical 
decisions [41, 42]. The apprehension of compromising their own clinical judgement and reasoning increases the reluctance of clinicians to adopt CDSS. Our results suggest that these perceptions may vary depending upon individual characteristics and type of healthcare setting. Experienced clinicians are less likely to use decision support tools due to fear of compromising established work practices and reducing autonomous control over processes and the content of clinical decisions [43]. On the other hand, younger clinicians tend to have better computer literacy and skills and so are more confident in using systems such as CDSS $[9,44]$. The likelihood of perceiving limited professional autonomy as a barrier to CDSS use was also identified in primary care. However, this might be due to the higher proportion of experienced clinicians (11 or more years) amongst our respondents that worked in primary care. Nonetheless, professional autonomy and freedom of clinical judgement have been identified in the literature as key factors to influence any kind of change process, specifically in primary care. Marjoribanks et al. (2003) indicated that concerns regarding clinical and financial freedom, and time constraints greatly contribute to hesitation towards a change process [45]. This again reiterates the need for flexibility in CDSS implementation to accommodate setting-specific circumstances and improve organisational support and infrastructure to improve CDSS adoption.

Clinicians' time and workflow constraints are significant factors that influence the adoption and use of CDSS in clinical settings. Our findings indicated that clinicians' time constraints and risk of workflow disruptions greatly contribute to end users' resistance to CDSS adoption. This is consistent with previous studies suggesting that failure to provide a fit between relevance, format and timeliness of recommendations negatively impact the uptake and utilisation of CDSS [11,46]. Moreover, our findings also highlight that in primary care there is a greater likelihood of perceiving time and workflow constraints as barriers to CDSS adoption. Despite the fact that workflow disruptions and time sensitivities are relevant to CDSS implementation across healthcare settings, factors such as assessing overwhelming amount of data within short visits and high workloads might contribute to limited CDSS adoption in primary care settings [47]. Further research is required to better understand the dynamics of time and workflow constraints for CDSS adoption in different care settings.

The risk and liability of malpractice was identified as an important barrier to uptake of CDSS. The goal of implementing CDSS is to ensure improved safety and quality of care and minimise the risk of adverse events. However, in the unlikely event of any harm to the patient, the legal and medical liability remains unclear [48]. Increased access to clinical information through CDSS, makes clinicians liable to use this information for decision-making. Studies have indicated that clinicians are usually concerned about excessive dependence on an external device or service, even when they believe that the knowledge base of systems may be rooted in experts' professional opinion rather than objective medical evidence [49]. This further contributes to uncertainty regarding the ethical and legal repercussions of overriding or ignoring CDSS alerts given that it can be categorised as professional negligence. Similarly, excessive and irrelevant alerts instead of adding any value to quality of care, increase the reluctance of end users to adopt systems to avoid any such liability as a consequence of alert fatigue [50-52]. 
Our study has several limitations. Because of low participation rates, the results may not be representative of knowledge and perceptions of Australian clinicians. Although the response rate was low, it is not dissimilar to other surveys conducted with healthcare practitioners; however, it may be that participants may have self-selected as a consequence of polarised views on the topic, introducing selection bias. Another limitation is the design of this survey, and perceptions of respondents of factors related to CDSS which may not correlate with actual practice. We allowed open responses to some questions which may have mitigated this to some extent. Future research is required to better understand underlying factors that shape users' perception about CDSS.

\section{Conclusions}

The organisational environment along with users' perceptions significantly influence CDSS adoption in healthcare settings. Users' reluctance stems from a lack of flexibility and adaptability of systems to users' requirements. The effective diffusion of CDSS in healthcare settings demands effective re-orientation of organisations to establish a facilitating and supportive environment for the uptake of CDSS. This includes providing support and training for users as well as involving end-users of varying levels of seniority in the development and implementation of the systems. Systems need to better reflect user requirements and setting dynamics to reduce end-user resistance to system uptake. These findings may help policy makers to develop a framework for sustainable and successful implementation of CDSS.

\section{Abbreviations}

CDSS

Clinical Decision Support System

UTAUT

Unified Theory of Acceptance and Use of Technology

\section{Declarations}

\section{Ethics approval and consent to participate}

This study was approved by The University of Adelaide Human Research Ethics Committee (H-2019-094)

\section{Consent for publication}

Not applicable.

\section{Availability of data and materials}

The survey data is not publicly available due to restricted data consent provided by participants concerning sharing of information. A limited dataset is available from the corresponding author on request. 


\section{Competing interests}

The authors declare that they have no competing interests.

\section{Funding}

This study was carried out as part of our routine work. M.L. is a recipient of an Adelaide Scholarship International (ASI).

\section{Authors' contributions}

$M L, A M$ and TM developed the conceptual framework and research protocol for the study. ML performed the analysis and drafted the manuscript. All authors contributed to interpretation of the analyses, critical revision of the manuscript and gave final approval of the version to be published.

\section{Acknowledgements}

The authors would like to thank Royal Australian Colleges of Surgeons, Physicians and General Practitioners, Rural Doctors Association of Australia and local health networks all over the Australia for their assistance in dissemination of the survey. We greatly appreciate the contribution of all the healthcare professionals who responded to our survey.

\section{References}

1. Sugden R, Kelly R, Davies S. Combatting antimicrobial resistance globally. Nature microbiology. 2016;1(10):16187.

2. Prestinaci F, Pezzotti P, Pantosti A. Antimicrobial resistance: a global multifaceted phenomenon. Pathog Glob Health. 2015;109(7):309-18.

3. Forrest GN, Van Schooneveld TC, Kullar R, Schulz LT, Duong P, Postelnick M. Use of electronic health records and clinical decision support systems for antimicrobial stewardship. Clin Infect Dis. 2014;59(Suppl 3):122-33.

4. Flynn HW, Batra NR, Schwartz SG, Grzybowski A. Antibiotic Stewardship. In: Endophthalmitis in Clinical Practice. Cham: Springer International Publishing; 2018. pp. 171-3.

5. Freundlich RE, Wanderer JP, Ehrenfeld JM. Clinical Decision Support Tools Need to Improve More Than Just Process Outcomes. Anesthesiology. 2018;129(3):614-4.

6. Jaspers MW, Smeulers M, Vermeulen H, Peute LW. Effects of clinical decision-support systems on practitioner performance and patient outcomes: a synthesis of high-quality systematic review findings. J Am Med Inform Assn. 2011;18(3):327-34.

7. Curtis CE, Al Bahar F, Marriott JF. The effectiveness of computerised decision support on antibiotic use in hospitals: A systematic review. PLoS One. 2017;12(8):e0183062. 
8. Simões A, Maia M, Gregório J, Couto I, Asfeldt A, Simonsen G, Póvoa P, Viveiros M, Lapão L.

Participatory implementation of an antibiotic stewardship programme supported by an innovative surveillance and clinical decision-support system. J Hosp Infect. 2018;100(3):257-64.

9. Varonen H, Kortteisto T, Kaila M, Group ES. What may help or hinder the implementation of computerized decision support systems (CDSSs): a focus group study with physicians. Fam Pract. 2008;25(3):162-7.

10. Kortteisto T, Komulainen J, Mäkelä M, Kunnamo I, Kaila M. Clinical decision support must be useful, functional is not enough: a qualitative study of computer-based clinical decision support in primary care. BMC Health Serv Res. 2012;12(1):349.

11. Moxey A, Robertson J, Newby D, Hains I, Williamson M, Pearson S-A. Computerized clinical decision support for prescribing: provision does not guarantee uptake. J Am Med Inform Assn. 2010;17(1):25-33.

12. Liberati EG, Ruggiero F, Galuppo L, Gorli M, González-Lorenzo M, Maraldi M, Ruggieri P, Polo Friz $H$, Scaratti G, Kwag KH, et al. What hinders the uptake of computerized decision support systems in hospitals? A qualitative study and framework for implementation. Implement Sci. 2017;12(1):113.

13. Venkatesh V, Thong JY, Xu X. Unified theory of acceptance and use of technology: A synthesis and the road ahead. J Assoc Inf Syst. 2016;17(5):328-76.

14. Venkatesh V, Thong JY, Xu X. Consumer acceptance and use of information technology: extending the unified theory of acceptance and use of technology. MIS quarterly. 2012;36(1):157-78.

15. Goh WP, Tao X, Zhang J, Yong J. Decision support systems for adoption in dental clinics: A survey. Knowl-Based Syst. 2016;104:195-206.

16. Esmaeilzadeh P, Sambasivan M, Kumar N, Nezakati H. Adoption of clinical decision support systems in a developing country: Antecedents and outcomes of physician's threat to perceived professional autonomy. Int J Med Inform. 2015;84(8):548-60.

17. Short D, Frischer M, Bashford J. Barriers to the adoption of computerised decision support systems in general practice consultations: a qualitative study of GPs' perspectives. Int J Med Inform. 2004;73(4):357-62.

18. Zheng K, Padman R, Johnson MP, Diamond HS. Understanding technology adoption in clinical care: Clinician adoption behavior of a point-of-care reminder system. Int J Med Inform. 2005;74(7):53543.

19. Rigopoulos G, Psarras J, Askounis D. A TAM model to evaluate user's attitude towards adoption of decision support systems. Res J Appl Sci. 2008;8(5):899-902.

20. Devaraj S, Sharma SK, Fausto DJ, Viernes S, Kharrazi H. Barriers and facilitators to clinical decision support systems adoption: A systematic review. J Bus Adm Res. 2014;3(2):p36.

21. Corbin J, Strauss A: Basics of qualitative research: Techniques and procedures for developing grounded theory: Sage publications; 2014.

22. Aitken C, Power R, Dwyer R. A very low response rate in an on-line survey of medical practitioners. Aust NZ J Publ Heal. 2008;32(3):288-9. 
23. Cho YI, Johnson TP, VanGeest JB. Enhancing surveys of health care professionals: a meta-analysis of techniques to improve response. Eval Health Prof. 2013;36(3):382-407.

24. Taylor T, Scott A. Do Physicians Prefer to Complete Online or Mail Surveys? Findings From a National Longitudinal Survey. Eval Health Prof. 2019;42(1):41-70.

25. Basedow M, Runciman WB, Lipworth W, Esterman A. Australian general practitioner attitudes to clinical practice guidelines and some implications for translating osteoarthritis care into practice. Aust J Prim Health. 2016;22(5):403-8.

26. Joyce CM, Scott A, Jeon S-H, Humphreys J, Kalb G, Witt J, Leahy A. The" Medicine in Australia: Balancing Employment and Life (MABEL)" longitudinal survey-Protocol and baseline data for a prospective cohort study of Australian doctors' workforce participation. BMC Health Serv Res. 2010;10(1):50.

27. Lugtenberg M, Weenink J-W, van der Weijden T, Westert GP, Kool RB. Implementation of multipledomain covering computerized decision support systems in primary care: a focus group study on perceived barriers. BMC Med Inform Decis Mak. 2015;15(1):82.

28. Wilson A, Opolski M. Identifying barriers to implementing a cardiovascular computerised decision support system (CDSS): a Delphi survey. J Innov Health Inform 2009:11.

29. Trivedi MH, Kern J, Marcee A, Grannemann B, Kleiber B, Bettinger T, Altshuler K, McClelland A. Development and implementation of computerized clinical guidelines: barriers and solutions. Methods Inf Med. 2002;41(05):435-42.

30. Lai F, Macmillan J, Daudelin DH, Kent DM. The Potential of Training to Increase Acceptance and Use of Computerized Decision Support Systems for Medical Diagnosis. Hum Factors. 2006;48(1):95108.

31. Tsiknakis $M$, Kouroubali A. Organizational factors affecting successful adoption of innovative eHealth services: a case study employing the FITT framework. Int J Med Inform. 2009;78(1):39-52.

32. Chan T, Brew S. Overcoming the barriers to using information systems. Nurs Times. 2004;100(49):44-6.

33. Yusof MM, Kuljis J, Papazafeiropoulou A, Stergioulas LK. An evaluation framework for Health Information Systems: human, organization and technology-fit factors (HOT-fit). Int J Med Inform. 2008;77(6):386-98.

34. Delone WH, Mclean ER. Measuring e-commerce success: Applying the DeLone \& McLean information systems success model. Int J Electron Comme. 2004;9(1):31-47.

35. Short D, Frischer M, Bashford J. The development and evaluation of a computerised decision support system for primary care based upon 'patient profile decision analysis'. J Innov Health Inform 2003:8.

36. Lugtenberg M, Pasveer D, van der Weijden T, Westert GP, Kool RB. Exposure to and experiences with a computerized decision support intervention in primary care: results from a process evaluation. BMC Fam Pract. 2015;16(1):141.

37. Gagnon M-P, Desmartis M, Labrecque M, Car J, Pagliari C, Pluye P, Frémont P, Gagnon J, Tremblay N, Légaré $F$. Systematic review of factors influencing the adoption of information and communication 
technologies by healthcare professionals. J Med Syst. 2012;36(1):241-77.

38. Venkatesh V, Morris MG, Davis GB, Davis FD. User Acceptance of Information Technology: Toward a Unified View. MIS Q. 2003;27(3):425-78.

39. Khairat S, Marc D, Crosby W, Al Sanousi A. Reasons For Physicians Not Adopting Clinical Decision Support Systems: Critical Analysis. JMIR Med Inform. 2018;6(2):e24-4.

40. Shibl R, Lawley M, Debuse J. Factors influencing decision support system acceptance. Decis Support Syst. 2013;54(2):953-61.

41. Goud R, van Engen-Verheul M, de Keizer NF, Bal R, Hasman A, Hellemans IM, Peek N. The effect of computerized decision support on barriers to guideline implementation: A qualitative study in outpatient cardiac rehabilitation. Int J Med Inform. 2010;79(6):430-7.

42. Cabana MD, Rand CS, Powe NR, Wu AW, Wilson MH, Abboud PA, Rubin HR. Why don't physicians follow clinical practice guidelines? A framework for improvement. JAMA. 1999;282(15):1458-65.

43. De Grood C, Raissi A, Kwon Y, Santana MJ. Adoption of e-health technology by physicians: a scoping review. J Multidiscip Healthc. 2016;9:335.

44. Leslie SJ, Hartswood M, Meurig C, McKee SP, Slack R, Procter R, Denvir MA. Clinical decision support software for management of chronic heart failure: Development and evaluation. Comput Biol Med. 2006;36(5):495-506.

45. Marjoribanks T, Lewis JM. Reform and autonomy: perceptions of the Australian general practice community. Soc Sci Med. 2003;56(10):2229-39.

46. Belard A, Buchman T, Forsberg J, Potter BK, Dente CJ, Kirk A, Elster E. Precision diagnosis: a view of the clinical decision support systems (CDSS) landscape through the lens of critical care. J Clin Monit Comput. 2017;31(2):261-71.

47. Hor CP, O'Donnell JM, Murphy AW, O'Brien T, Kropmans TJB. General practitioners' attitudes and preparedness towards Clinical Decision Support in e-Prescribing (CDS-eP) adoption in the West of Ireland: a cross sectional study. BMC Med Inform Decis Mak. 2010;10:2-2.

48. Ridgely MS, Greenberg MD: Too many alerts, too much liability: sorting through the malpractice implications of drug-drug interaction clinical decision support. Louis UJ Health L \& Pol'y 2011, 5:257.

49. Fox J, Thomson R: Clinical decision support systems: a discussion of quality, safety and legal liability issues. In: Proceedings of the AMIA Symposium: 2002: American Medical Informatics Association; 2002: 265.

50. Berner ES. Ethical and legal issues in the use of clinical decision support systems. J Healthc Inf Manag. 2002;16(4):34-7.

51. Mangalmurti SS, Murtagh L, Mello MM. Medical Malpractice Liability in the Age of Electronic Health Records. Survey Anesthesiol. 2011;55(6):317-9.

52. Kesselheim AS, Cresswell K, Phansalkar S, Bates DW, Sheikh A. Clinical decision support systems could be modified to reduce 'alert fatigue'while still minimizing the risk of litigation. Health Aff. 2011;30(12):2310-7. 


\section{Tables}

Due to technical limitations, Tables are available in supplementary section.

\section{Figures}

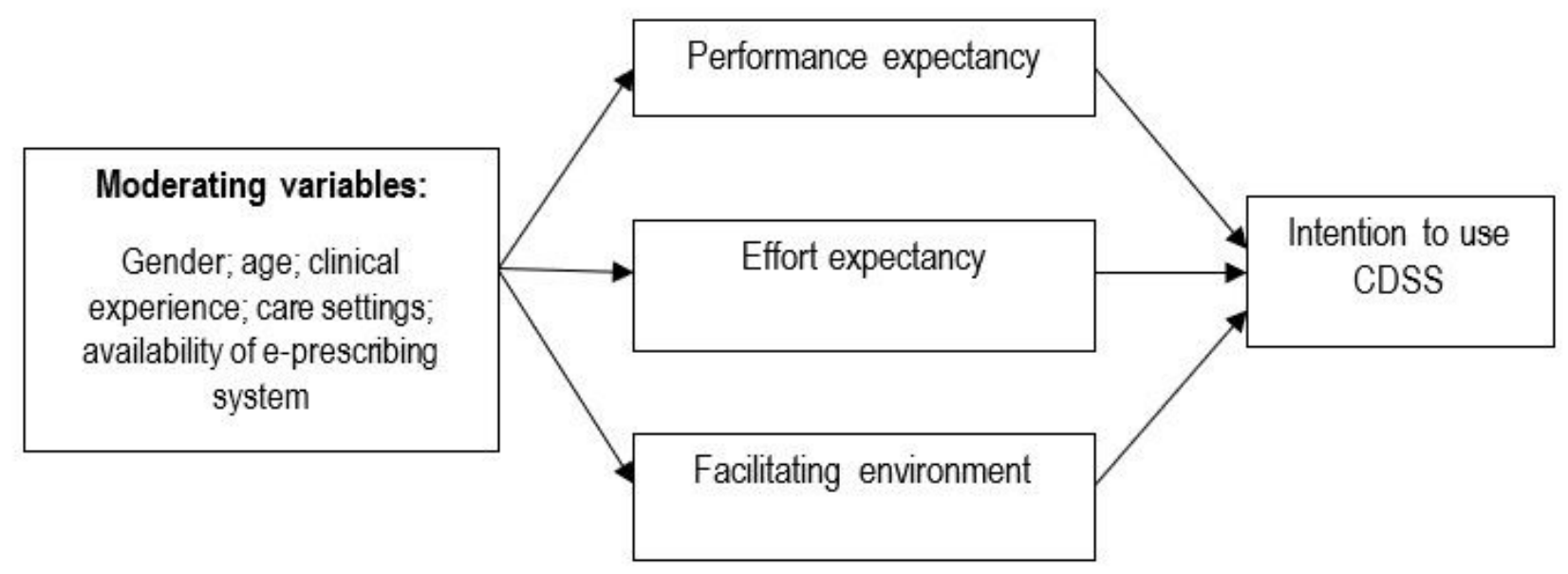

\section{Figure 1}

Theoretical framework (UTAUT model) of the study

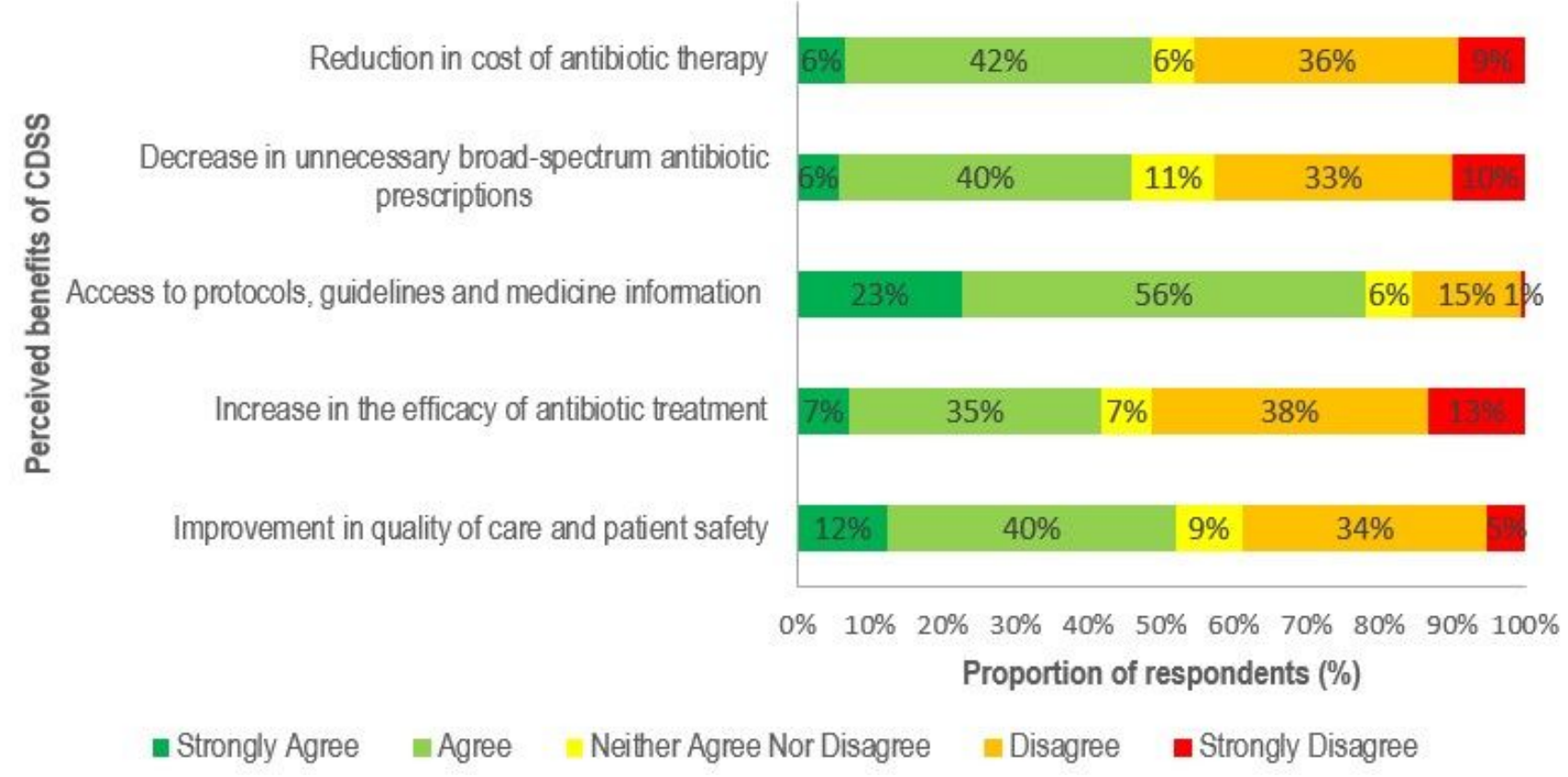

Figure 2 


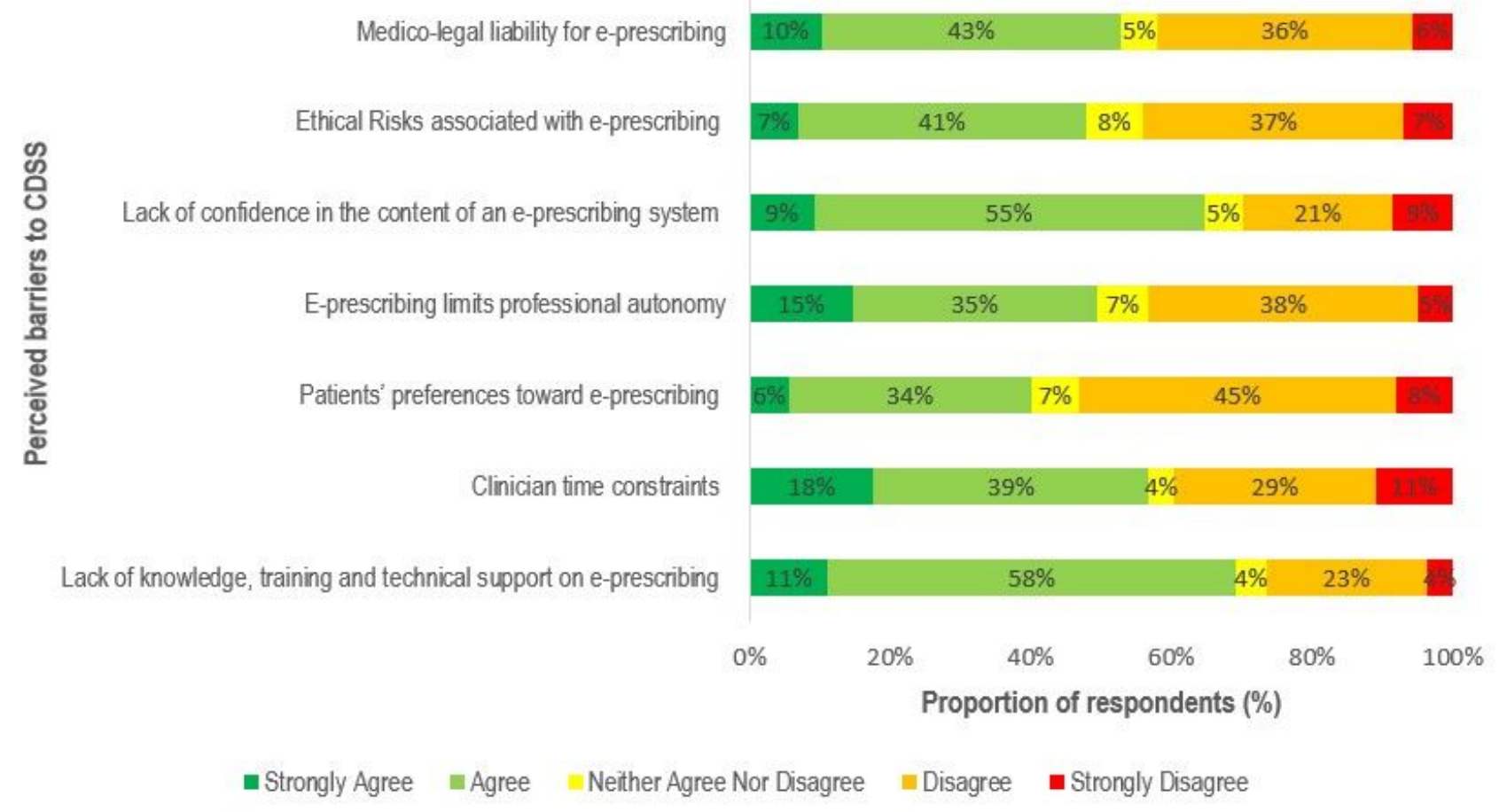

\section{Figure 3}

Reported barriers to CDSS adoption.

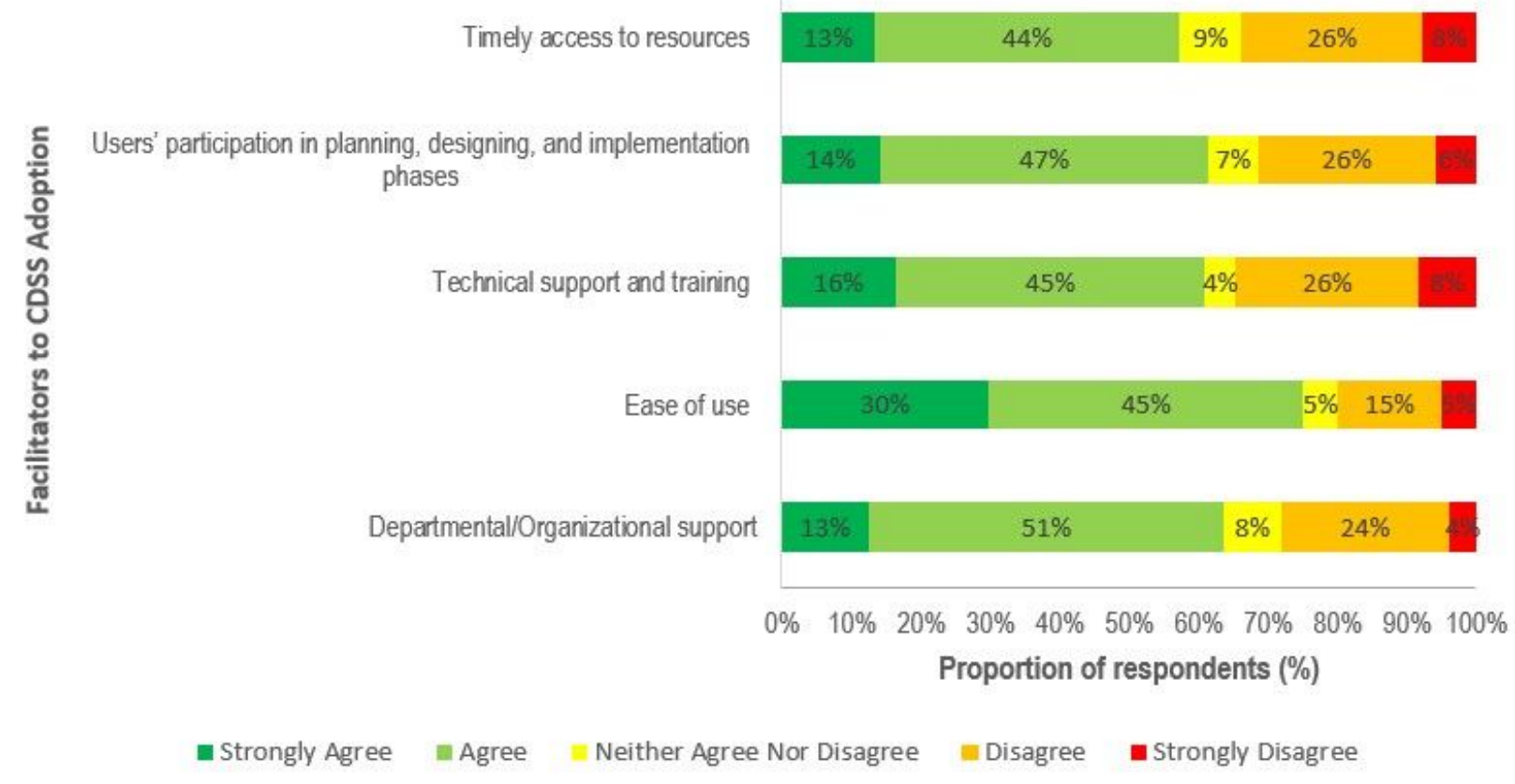


Figure 4

Facilitators to CDSS adoption

\section{Supplementary Files}

This is a list of supplementary files associated with this preprint. Click to download.

- Table4.jpg

- Table3.jpg

- Supplementaryfile1Surveyprimarycare.docx

- Supplementaryfile2Surveyhospitalsettings.docx

- Table2.jpg 